\title{
More about arc-polarized structures in the solar wind
}

\author{
S. Haaland ${ }^{1,2}$, B. Sonnerup ${ }^{3}$, and G. Paschmann ${ }^{4}$ \\ ${ }^{1}$ Max-Planck-Institut für Sonnensystemforschung, Katlenburg-Lindau, Germany \\ ${ }^{2}$ Department of Physics and Technology, University of Bergen, Norway \\ ${ }^{3}$ Thayer School of Engineering, Dartmouth College, Hanover, New Hampshire, USA \\ ${ }^{4}$ Max-Planck-Institut für extraterrestrische Physik, Garching, Germany \\ Correspondence to: S. Haaland (stein.haaland@ift.uib.no)
}

Received: 3 January 2012 - Revised: 1 May 2012 - Accepted: 7 May 2012 - Published: 25 May 2012

\begin{abstract}
We report results from a Cluster-based study of the properties of 28 arc-polarized magnetic structures (also called rotational discontinuities) in the solar wind. These Alfvénic events were selected from the database created and analyzed by Knetter (2005) by use of criteria chosen to eliminate ambiguous cases. His studies showed that standard, four-spacecraft timing analysis in most cases lacks sufficient accuracy to identify the small normal magnetic field components expected to accompany such structures, leaving unanswered the question of their existence. Our study aims to break this impasse. By careful application of minimum variance analysis of the magnetic field (MVAB) from each individual spacecraft, we show that, in most cases, a small but significantly non-zero magnetic field component was present in the direction perpendicular to the discontinuity. In the very few cases where this component was found to be large, examination revealed that MVAB had produced an unusual and unexplained orientation of the normal vector. On the whole, MVAB shows that many verifiable rotational discontinuities $\left(B_{n} \neq 0\right)$ exist in the solar wind and that their eigenvalue ratio (EVR $=$ intermediate/minimum variance) can be extremely large (up to EVR =400). Each of our events comprises four individual spacecraft crossings. The events include 17 ionpolarized cases and 11 electron-polarized ones. Fifteen of the ion events have widths ranging from 9 to 21 ion inertial lengths, with two outliers at 46 and 54. The electronpolarized events are generally thicker: nine cases fall in the range 20-71 ion inertial lengths, with two outliers at 9 and 13. In agreement with theoretical predictions from a onedimensional, ideal, Hall-MHD description (Sonnerup et al., 2010), the ion-polarized events show a small depression in field magnitude, while the electron-polarized ones tend to show a small enhancement. This effect was also predicted
\end{abstract}

by Wu and Lee (2000). Judging only from the sense of the plasma flow across our DDs, their propagation appears to be sunward as often as anti-sunward. However, we argue that this result can be misleading as a consequence of the possible presence of magnetic islands within the DDs. How the rotational discontinuities come into existence, how they evolve with time, and what roles they play in the solar wind remain open questions.

Keywords. Interplanetary physics (Discontinuities; Solar wind plasma; Instruments and techniques)

\section{Introduction}

It has been known since the 1960s that solar wind carries with it an abundance of directional discontinuities (DDs), across which the magnetic field and the plasma flow and other plasma parameters can change dramatically. In the early observations, the structure of these DDs could usually not be resolved due to limitations in the instrumentation, but today their magnetic structure (and less often their plasma structure) can readily be measured. Among all of these DDs, there is a small subset with the property that the field magnitude remains approximately constant as one crosses the DD layer, and the plasma density and pressure remain about the same. Such discontinuities are believed to be large amplitude Alfvén waves. They are thin layers but are thought to be extended surfaces in space. They are assumed to propagate in a direction perpendicular to themselves along a small component of the magnetic field in the direction normal to the surface. In other words, the regions on the two sides of this type of DD are magnetically connected and there is an associated plasma flow from one side of the layer to the other. In 
the proper (co-moving) frame of the discontinuity, this flow is either parallel or antiparallel to the magnetic field, and it is approximately Alfvénic. In MHD parlance, these discontinuities are rotational discontinuities (RDs), but in solar wind studies they are also referred to as "arc-polarized" structures. How these structures are formed and evolve in the solar wind plasma has been intensely studied but is not yet well understood. One possibility is that they develop when large amplitude, plane-polarized Alfvén waves, generated near the sun, are transported outward in the solar wind (Vasquez and Hollweg, 1996).

In this paper we describe and analyze 28 encounters of arc-polarized structures by the four Cluster spacecraft in the near-Earth solar wind. The paper is a direct continuation of our earlier study (Sonnerup et al., 2010), hereafter referred to as Paper I, in which the focus was on a particular kind of arc structure, in which the magnetic field was seen to rotate, first in one sense, and then back again in the other sense. We called these structures "double-arc polarized". In the present article we focus on single-arc structures but include the two branches of our previous double-arc event as separate events. We will determine the thickness of the 28 discontinuities, their normal magnetic field component and their sense of polarization. We will also examine the small deviations from constancy of the total magnetic field, seen in the events, and how they depend on the sense of polarization. We will present evidence that these features require mathematical description in terms of Hall MHD, a result that at first seems anomalous, because the discontinuity thicknesses extend over many ion inertial lengths, suggesting that ordinary MHD should suffice.

The paper is organized as follows. In Sect. 2, we briefly summarize the theoretical results developed in Paper I and present lowest-order approximate formulas that help in the understanding of those results. In Sect. 3, we present our data set and the procedure used to identify the 28 events in our study. In Sect. 4, we describe our data analysis procedures and give sample illustrations of the events. In Sect. 5, we present the essential results for each event in two tables: one containing base information and the other the results of minimum variance analysis of the magnetic field (MVAB). The goal is to determine the magnetic field component along the direction normal to the layer, taken to be the minimum variance direction. Section 6 contains a summary of the most important findings and further discussion of them. Secondorder approximate formulas for certain theoretical hodogram properties are given in Appendix A. Finally, theoretical results for arc-polarized discontinuities (RDs) having large variations in field magnitude are presented in Appendix B.

\section{Theoretical background}

The results of the data analysis will be interpreted in the context of the Hall-MHD description of arc-polarized structures

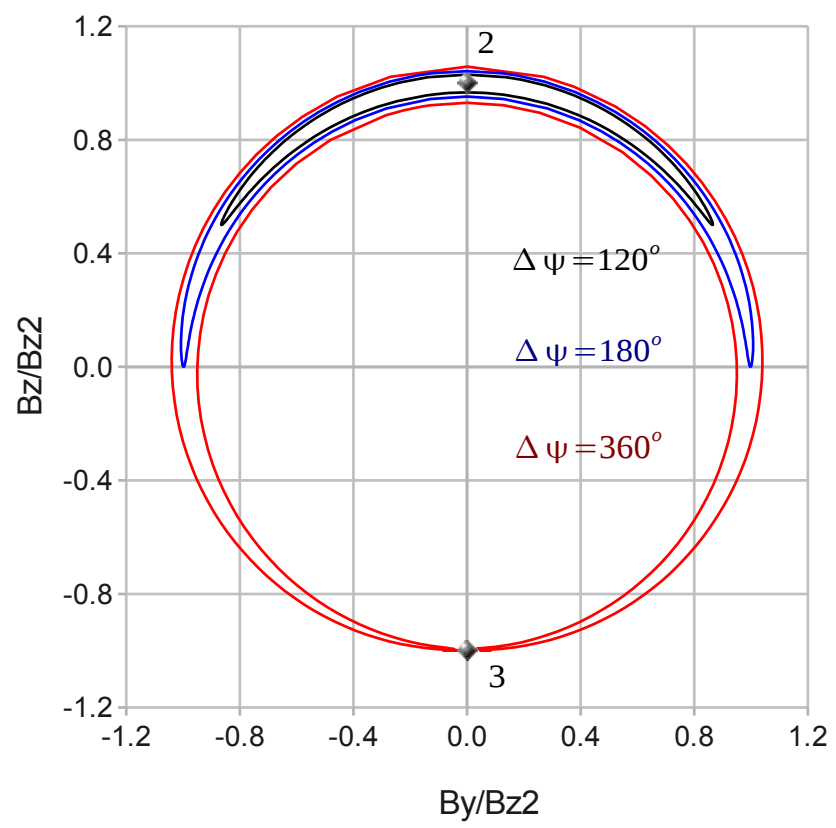

Fig. 1. Hodogram traces for $A_{x 2}^{2}=1.001, \beta_{2}=1, \gamma=2$, and $\sin ^{2} \theta_{2}=0.996$ and with three values of the net rotation angle, $\Delta \Psi=2 \Psi^{*}$, of the tangential field. Fixed Point 2 ( a "center") is at the center of the nest of traces $\left(B_{\mathrm{y}} / B_{\mathrm{z} 2}=0 ; B_{\mathrm{z}} / B_{\mathrm{z} 2}=1\right)$; Fixed Point 3 (a saddle point) is on the negative $B_{\mathrm{Z}}$-axis, at the bottom of the plot, where the red curves intersect. (From Sonnerup et al., 2010).

(rotational discontinuities) in the solar wind, developed in Paper I. That Hall MHD is needed was made clear already in the work by $\mathrm{Wu}$ and Lee (2000), although the numerical examples they give are intermediate shocks (with a tangential field rotation of exactly $180^{\circ}$ ) rather than rotational discontinuities (for which the rotation angle is usually $<180^{\circ}$ ). However, these two types of structures are closely related and the conclusion reached by $\mathrm{Wu}$ and Lee that electron-polarized discontinuities should exhibit a field maximum within their structure, while the ion-polarized ones should exhibit a field minimum, remains valid for both types.

In mathematical terms, an intermediate shock is a transition from one fixed point (the upstream state) of the system of equations to another one (the downstream state), and it requires the presence of some dissipative process. In the absence of dissipation, no transition from one fixed point to another is possible but only infinite wave trains involving a gyration around a fixed point or a solitary wave in which the upstream and downstream states are identical and the tangential field rotation is $360^{\circ}$. An example, taken from Paper I, of the behavior of the tangential magnetic field in such dissipationfree structures is shown in Fig. 1. In this figure, a fixed point, denoted by the numeral 2 and located at $B_{\mathrm{y}}=0 ; B_{\mathrm{z}} / B_{\mathrm{z} 2}=1$, is at the center of a nest of magnetic hodograms showing rotation back and forth of the tangential field, by $120^{\circ}$ (black curve), by $180^{\circ}$ (blue curve), and by $360^{\circ}$ (red curve). The 
red curves begin and end at a second fixed point, denoted by the numeral 3. They represent the aforementioned solitary waves. The upper branch of each curve is electron polarized and the lower branch is ion polarized; the figure illustrates the occurrence of a field maximum on the former branch and a field minimum on the latter. In the absence of dissipation, Point 2 is a "center", as shown in the figure; if dissipation is included, it is converted to an unstable spiral point and then serves as the upstream state of one type of intermediate shock. The fixed Point 3 is a saddle point and serves as the downstream state of a weak intermediate shock (and also as the upstream state of a slow shock) (for details, see Hau and Sonnerup, 1990). Since the coplanarity condition for shocks requires all four fixed points of the system of equations to be located on a straight line through the origin, here chosen to be the $B_{\mathrm{Z}}$-axis, and since Point 2 (positive $B_{\mathrm{Z}}$ ) and Point 3 (negative $B_{\mathrm{Z}}$ ) are on opposite sides of the origin, the intermediate shock contains a tangential field rotation of exactly $180^{\circ}$, in either the electron sense or in the ion sense. Examples of such structures may be found in Fig. 5 of the article by Hau and Sonnerup (1990) or in Figs. 4 and 5 of the article by $\mathrm{Wu}$ and Lee (2000). Not shown in Fig. 1 is fixed Point 1, on the positive $B_{\mathrm{Z}}$-axis, which is the upstream state of the fast shock (and also of superfast, weak and strong, intermediate shocks) and fixed Point 4 , on the negative $B_{\mathrm{Z}}$-axis, which is the downstream state of the slow shock (and also of strong intermediate shocks).

The hodogram in Fig. 1 represents an infinite wave train in which the tip of the tangential field vector moves periodically from side to side along the banana-shaped hodogram curve in either the anticlockwise or the clockwise sense. The former sense occurs when the normal flow velocity $v_{\mathrm{X}}$ (in the wave frame) and the normal field component $B_{\mathrm{X}}$ have the same sign, the latter when they have opposite signs. In Paper I, a full period of this motion was used to account for observed "double-arc" polarized structures sometimes seen in the solar wind. In the present paper, one of the branches of the hodogram, either the upper or the lower one, will be used to account for observed "single-arc" polarized structures (RDs). In what follows, the $\mathrm{x}$-axis and $\boldsymbol{n}$, the unit vector normal to the discontinuity, are taken to be parallel and pointing sunward.

Details of the mathematical model on which Fig. 1 is based are given in Paper I. There, it is shown that, for the structures of interest to us, the Alfvén number $A_{\mathrm{x}} \equiv v_{\mathrm{x}} \sqrt{\mu_{0} \rho} / B_{\mathrm{x}}$, based on the flow velocity component $v_{\mathrm{x}}$ and field component $B_{\mathrm{X}}$ in the normal direction, has magnitude slightly greater than one, when evaluated at Point 2. For example, we have $A_{\mathrm{x} 2}^{2}=1.001$ in Fig. 1. It is also shown that the locus of points in the hodogram plane where $A_{\mathrm{x}}^{2}=1$ is a circle with its center very slightly displaced upward from the origin $\left(B_{\mathrm{y}}=B_{\mathrm{Z}}=0\right)$ and that this "critical" circle marks the locus of the two turning points of the crescent-shaped hodogram curves, where $B_{\mathrm{Z}}$ reaches its minimum value. Outside the critical circle, we have $A_{\mathrm{x}}^{2}>1$ and the hodogram curve located there (the upper branch in Fig. 1) is electron polarized. The lower branch of the hodogram, which is located inside the critical circle, has $A_{\mathrm{x}}^{2}<1$ and is ion polarized. As argued in Paper I, this result is what is expected from the whistler dispersion relation: the electron (ion) whistler has phase speed greater (smaller) than the Alfvén speed $B_{\mathrm{x}} / \sqrt{\mu_{0} \rho}$. On the basis of the normal component (the x-component) of the stress balance, $\rho v_{\mathrm{x}}^{2}+p+\left(B_{\mathrm{y}}^{2}+B_{\mathrm{z}}^{2}\right) / 2 \mu_{0}=$ const., in combination with mass conservation, $\rho v_{\mathrm{x}}=$ const., and the polytropic law, $p / \rho^{\gamma}=$ const., it was further demonstrated that, as long as $\gamma>1$, the part of the hodogram where $A_{\mathrm{x}}^{2}>1$, i.e., the electron-polarized (upper) branch, must have larger field magnitudes than the ion-polarized (lower) branch, on which $A_{\mathrm{x}}^{2}<1$ : This is indeed the behavior seen in Fig. 1 . That the field increase is monotonical as one moves along the hodogram curve from the field minimum at $B_{\mathrm{y}}=0$ on the lower branch to first reach one of the turning points and then moves on to the field maximum at $B_{\mathrm{y}}=0$ on the upper branch is difficult to ascertain in the figure but is evident for some of the hodograms shown in Fig. B1. From the same considerations, it was also concluded that the separation between the two branches of the hodogram must become smaller as the ratio of effective specific heats, $\gamma=c_{\mathrm{p}} / c_{\mathrm{v}}$, approaches unity (for details, see item 2 in Sect. 5 of Paper I). Another consequence of the normal stress balance is that an electron-polarized DD must have a density and pressure minimum and an ion-polarized DD a density and pressure maximum in its middle, a conclusion first reached by Lee and Kan (1982) on the basis of a theoretical model that included a trapped electron population. Subsequently, the density effect has been found in a number of numerical simulations (for some of the references, see Wu and Lee, 2000).

Note that in the MHD limit the dispersive effects disappear. The critical circle $\left(A_{\mathrm{x}}^{2}=1\right)$, which is then centered exactly at the origin of the hodogram, represents the MHD limit of a rotational discontinuity, in which the field magnitude is precisely constant. The price paid for this simple behavior is that the field rotation in the discontinuity has become infinitely slow: The fixed Point 2 has degenerated to become the entire critical circle.

To quantitatively illustrate the behavior described above, we now assume that the quantity $\epsilon_{2}^{2}=\left(A_{\mathrm{x} 2}^{2}-1\right)$, where the subscript 2 denotes conditions at the fixed Point 2 , is much less than one (as it is in Fig. 1). By series expansion of the exact solution (Eqs. 4, 8, 9 and 13) in Paper I, we have then shown that, to lowest order in $\epsilon_{2}$, the values of $A_{\mathrm{x}}^{2}$ and the corresponding values of $B_{\mathrm{Z}} / B_{\mathrm{Z} 2}$ at the top of the outer and inner branches of the hodogram in the figure (where $B_{\mathrm{y}}=$ 0 , this location to be indicated by the subscript 0 ), and the corresponding spatial rotation rate of the tangential field are given by 


$$
\begin{aligned}
A_{\mathrm{x} 0}^{2} & =1 \pm 2 \epsilon_{2} \sin \left(\Psi^{*} / 2\right) / \sqrt{u_{2}} \\
B_{\mathrm{z} 0} / B_{\mathrm{z} 2} & =1 \pm 2 \epsilon_{2} \sqrt{u_{2}} \sin \left(\Psi^{*} / 2\right) \\
d\left(B_{\mathrm{y} 0} / B_{\mathrm{z} 2}\right) / d x & = \pm\left(2 \epsilon_{2} / \lambda_{i 2}\right) \sin \left(\Psi^{*} / 2\right) / \sqrt{u_{2}}
\end{aligned}
$$

where $\Psi^{*}=\tan ^{-1}\left(B_{\mathrm{y}} / B_{\mathrm{z}}\right)^{*}$ is the field rotation angle at the turning points (those points are denoted by an asterisk), so that the total rotation angle of the tangential field, from one turning point to the other, is $\Delta \Psi=2 \Psi^{*}$. Also, $B_{\mathrm{z} 2}$ and $\lambda_{i 2}$ are the tangential field component and the ion inertial length, evaluated at the fixed Point 2 , and the quantity $u_{2}$ is defined by

$u_{2} \equiv \frac{\gamma \beta_{2} / 2-\cos ^{2} \theta_{2}}{\sin ^{2} \theta_{2}}$

where $\theta_{2}=\tan ^{-1}\left(B_{\mathrm{z} 2} / B_{\mathrm{x}}\right)$ is the angle, at the fixed Point 2, between the total field and the vector $\hat{\boldsymbol{x}}$ normal to the structure (note that $u_{2}=1$ in Fig. 1). Also, $\beta_{2}$ is the ratio of plasma pressure to magnetic pressure at Point 2. Provided that $u_{2}>0$ and $\Psi^{*} \gg \epsilon_{2}$, Eqs. (1), (2), and (3) express the dominant behavior of the structures, which is seen to be of order $\epsilon_{2}$. Expressions that include order $\epsilon_{2}^{2}$ are given in Appendix A.

Equation (2) shows that the upper, electron-polarized, branch (described by the + sign) of the hodogram in Fig. 1 has a field maximum in its middle, while the lower, ionpolarized branch (described by the - sign) has a field minimum. These features should be present in the observations. In Fig. 1 for which $\epsilon_{2}^{2}=0.001$, this maximum or minimum amounts to less than $5 \%$ of the field magnitude at the fixed point 2 , but when $\epsilon_{2}$ is larger, substantial variations in field magnitude will occur (see Appendix B). The maximum separation of the two hodogram branches and the rate of field rotation at the top of the hodogram (where $B_{\mathrm{y}}=0$ ) both scale as $\epsilon_{2} \sin \left(\Psi^{*} / 2\right)$, i.e., they get smaller the closer $A_{\mathrm{x} 2}^{2}$ is to one and the smaller is the maximum rotation angle $\Psi^{*}$ of the field, away from the points where $B_{\mathrm{y}}=0$. As can be seen from Eqs. (2) and (3), with the expression for $u_{2}$ in Eq. (4), the branch separation and field rotation rate also get smaller as $\gamma$ and $\beta_{2}$ decrease.

Figure 4 of Paper I suggests that, for small values of $\epsilon_{2}$, the behavior of the local field angle, $\Psi=\tan ^{-1}\left(B_{\mathrm{y}} / B_{\mathrm{z}}\right)$ at points along the $\mathrm{x}$-axis may be approximated by $\Psi=\Psi^{*} \sin k x$, where $k$ can be evaluated by use of Eq. (3). The thickness of a single-arc discontinuity (an RD) then becomes

$d=\pi / k=\lambda_{i 2} \frac{\pi \sqrt{u_{2}}}{\epsilon_{2}} \frac{\Psi^{*} / 2}{\sin \left(\Psi^{*} / 2\right)}$.

This result illustrates that the thickness can be much larger than the ion inertial length, provided $\epsilon_{2}$ is sufficiently small. As mentioned already, it also shows that the thickness increases with increasing net rotation angle $2 \Psi^{*}$ (expressed in radians) and with increasing $\gamma$ and $\beta_{2}$. From Eq. (5) one can express $\epsilon_{2} / \sqrt{u_{2}}$ in terms of $d / \lambda_{i 2}$ and $\Psi^{*}$, both of which can be reasonably well determined from the measurements. This result can then be used to convert Eqs. (1), (2), and (3) to the forms

$$
\begin{aligned}
A_{\mathrm{x} 0}^{2} & =1 \pm\left(\lambda_{i 2} / d\right) \pi \Psi^{*} \\
B_{\mathrm{z} 0} / B_{\mathrm{z} 2} & =1 \pm u_{2}\left(\lambda_{i 2} / d\right) \pi \Psi^{*} \\
d\left(B_{\mathrm{y} 0} / B_{\mathrm{z} 2}\right) / d x & = \pm(\pi / d) \Psi^{*}
\end{aligned}
$$

In these expressions, the angle $\Psi^{*}$, which is half of the magnetic shear angle, is, as before, expressed in radians. In Eq. (4) for $u_{2}$, the plasma beta is reasonably well known, while the angle $\theta_{2}$ is known with an accuracy given by how well the normal magnetic field component $B_{\mathrm{x}}=B_{n}$ has been determined. Usually, this angle is close to $90^{\circ}$. Experience reported in Paper I indicates that $\gamma$ values considerably less than the isentropic value, $\gamma=5 / 3$, and approaching the isothermal value, $\gamma=1$, are needed.

A conceptual problem with the use of a full period of the infinite wave solution to represent a double-arc structure in Paper I or a half period to describe a single-arc structure (i.e., an RD) remains: The turning points are not fixed points of the system of equations, because, while $d B_{\mathrm{Z}} / d x=0$, the derivative $d B_{\mathrm{y}} / d x=O\left(\epsilon_{2}^{2}\right) \neq 0$ at the turning points. In a strict sense, the turning points in the hodogram can therefore not serve as the upstream or downstream state of a timeindependent, one-dimensional structure, described by ideal Hall MHD with isotropic pressure. But these points are very close to being fixed points; small deviations from the assumed model, caused by, for example, slow time evolution of the discontinuity thickness, or perhaps small deviations from pressure isotropy could allow them to serve as upstream and downstream states of an Alfvénic discontinuity. The net field rotation $\Delta \Psi=2 \Psi^{*}$ across such a single-arc structure is specified by the field orientations in the region upstream and downstream of the discontinuity. Since these two fields are usually not even approximately antiparallel, an intermediate shock or part of it cannot be used to describe the field transition from the upstream to the downstream state.

\section{Data set}

In our search for arc-polarized events, we started with the set of DDs identified by Thorsten Knetter in his thesis (Knetter, 2005), using high-resolution magnetic field measurements by the FGM instruments (Balogh et al., 1997) on the four Cluster spacecraft. As described in Knetter et al. (2004) and Knetter (2005), events were selected following the criteria first introduced by Burlaga (1969) and then used by Tsurutani and Smith (1979). These criteria essentially require magnetic field rotations $>30^{\circ}$ within a three-minute interval. Knetter then removed cases, where model calculations suggested a bow shock connection, as well as cases where MVAB gave eigenvalue ratios (EVRs) less than 2. Given that Cluster is never far from the bow shock, the avoidance of bow shock 
connection biases the data set toward cases where the DD normals have large GSE X-components (recall that by definition, the $\mathrm{X}$-component of our normal vectors are always positive).

Knetter's database comprises the years 2001-2003. For the year 2003, his selection criteria resulted in a total of 204 cases. We chose to examine the events from that year only, because of the larger $(\approx 5000 \mathrm{~km})$ inter-spacecraft separations. The requirement for availability of plasma moments from the CIS instrument (Rème et al., 1997) further reduced the number of cases to 190 . Our investigation was greatly facilitated by Thorsten Knetter providing us, not only with the crossing times, but also with many other results from his analysis, including lists of the DD normal directions and speeds, obtained from four-point timing analysis, the normal directions and eigenvalue ratios (EVRs) from standard MVAB for all four spacecraft, and the shear angle of the field.

To find single-arc candidates, we pre-filtered the event list for cases where Knetter's analysis had shown the EVRs for spacecraft $\mathrm{C} 1$ to be $\geq 5$ and the field shear angle to be $\geq 55^{\circ}$. From high-resolution magnetic field plots, we then selected those cases where $B$ was constant to within $1 \mathrm{nT}$. We also required that Walén relation tests (see below) performed on the data set give slope magnitudes $\geq 0.70$. This left us with 26 events, of which two were of the double-arc kind, for detailed analysis.

\section{Analysis}

The most important properties characterizing single-arc structures are their normal magnetic field component, their polarization, and their propagation sense, either away from the sun or toward it. To establish these properties and to characterize the plasma conditions, we used the Cluster magnetic field and plasma ion and electron data, obtained from the Cluster Active Archive (CAA), as well as plasma ion data from the solar wind monitor ACE, obtained from CDAWeb.

\subsection{Minimum variance analysis}

A key element to be established for the arc-polarized cases is that they should have a finite $\boldsymbol{B}_{n}$. As demonstrated in two seminal studies (Knetter et al., 2004; Knetter, 2005), the DDs observed by Cluster have $B_{n}$ magnitudes that are very small when normal directions, obtained by triangulation based on standard four-point timing analysis, are used, hardly ever exceeding $20 \%$ of the total field. The triangulation technique has also been referred to as the constant velocity approach, CVA (see, e.g., Haaland et al., 2004). This result is in strong disagreement with results from MVAB where large normal components are often found, unless the ratio of intermediate to minimum eigenvalue (EVR) of the magnetic variance matrix is quite large. In Paper I we have presented such a case, which demonstrates that, for large EVR, careful use of
MVAB can yield small, but reliable $B_{n}$ values. In CVA, such small values tend to be obscured by the assumptions of onedimensionality and constant DD velocity, as well as by uncertainties in the crossing times (Vogt et al., 2011). In order to automate the analysis, we developed a novel procedure for selecting the optimal data interval for MVAB, as described in the following section.

\subsubsection{Finding the optimal time segment for MVAB}

Minimum variance analysis will generally give different results (eigenvectors, eigenvalues) and statistical error bounds if the analysis window (center time and/or length of analysis interval, i.e., the "nest size") is changed (e.g., Sonnerup and Scheible, 1998). To determine the optimal time segment, we have therefore tested a large number of center times and analysis intervals, and then investigated how the eigenvalue ratio, the normal component of the magnetic field $\left(B_{n}\right)$, as well as the angular errors of the normal vector $\boldsymbol{n}$ and errors in the $B_{n}$ estimate are affected. Figure 2 shows, separately for $\mathrm{C} 1$ and $\mathrm{C} 3$, time series of the measured magnetic field, followed by color-coded 2-D maps of some of these quantities for one of the arc-polarized structures we investigated.

To construct these maps, we first get a rough estimate of the central time of the $\mathrm{DD}$, referred to as $T_{\mathrm{c} 0}$, by visual inspection of the time series of the high-resolution magnetic field data. We then perform MVAB on a set of successively larger time segments (referred to as a nest) around this center time. For each nest member, the intermediate-to-minimum eigenvalue ratio (EVR), the associated $B_{n}$, calculated from the normal direction and the average field vector $\boldsymbol{B}$, and the statistical error, $d B_{n}$, are all recorded and plotted in the respective maps. We used nest sizes between 0.4 and $10 \mathrm{~s}$, occasionally up to $20 \mathrm{~s}$, with a granularity of 2 samples of $46 \mathrm{~ms}$ duration, each time adding one extra sample on each side of the time segment. This gave a total of $N=102$ different nest members. We then shifted the center time, $T_{\mathrm{c}}$, and repeated this procedure $M=69$ times. The result is $N \times M=7038$ different combinations of data intervals and center times. In the figure, the horizontal axis of the maps represents the center times, and the vertical axis the length of the data intervals (labeled "nest sizes").

The $d B_{n}$ values were computed from Eqs. (8.23) and (8.24) in the review article by Sonnerup and Scheible (1998) that were derived by Khrabrov and Sonnerup (1998a). Their estimate (in radians) of the statistical portion of the angular errors in the normal direction is given by

$$
\begin{aligned}
\left|\Delta \varphi_{i j}\right| & =\left|\Delta \varphi_{j i}\right|=\left\langle\left\langle\left(\Delta x_{i j}\right)^{2}\right\rangle\right\rangle^{1 / 2}=\left\langle\left\langle\left(\Delta x_{j i}\right)^{2}\right\rangle\right\rangle^{1 / 2} \\
& =\sqrt{\frac{\lambda_{3}}{(M-1)} \frac{\left(\lambda_{\mathrm{i}}+\lambda_{j}-\lambda_{3}\right)}{\left(\lambda_{\mathrm{i}}-\lambda_{j}\right)^{2}}}, i \neq j .
\end{aligned}
$$

Based on these angular errors, the statistical uncertainty in the normal component of the average magnetic field becomes 

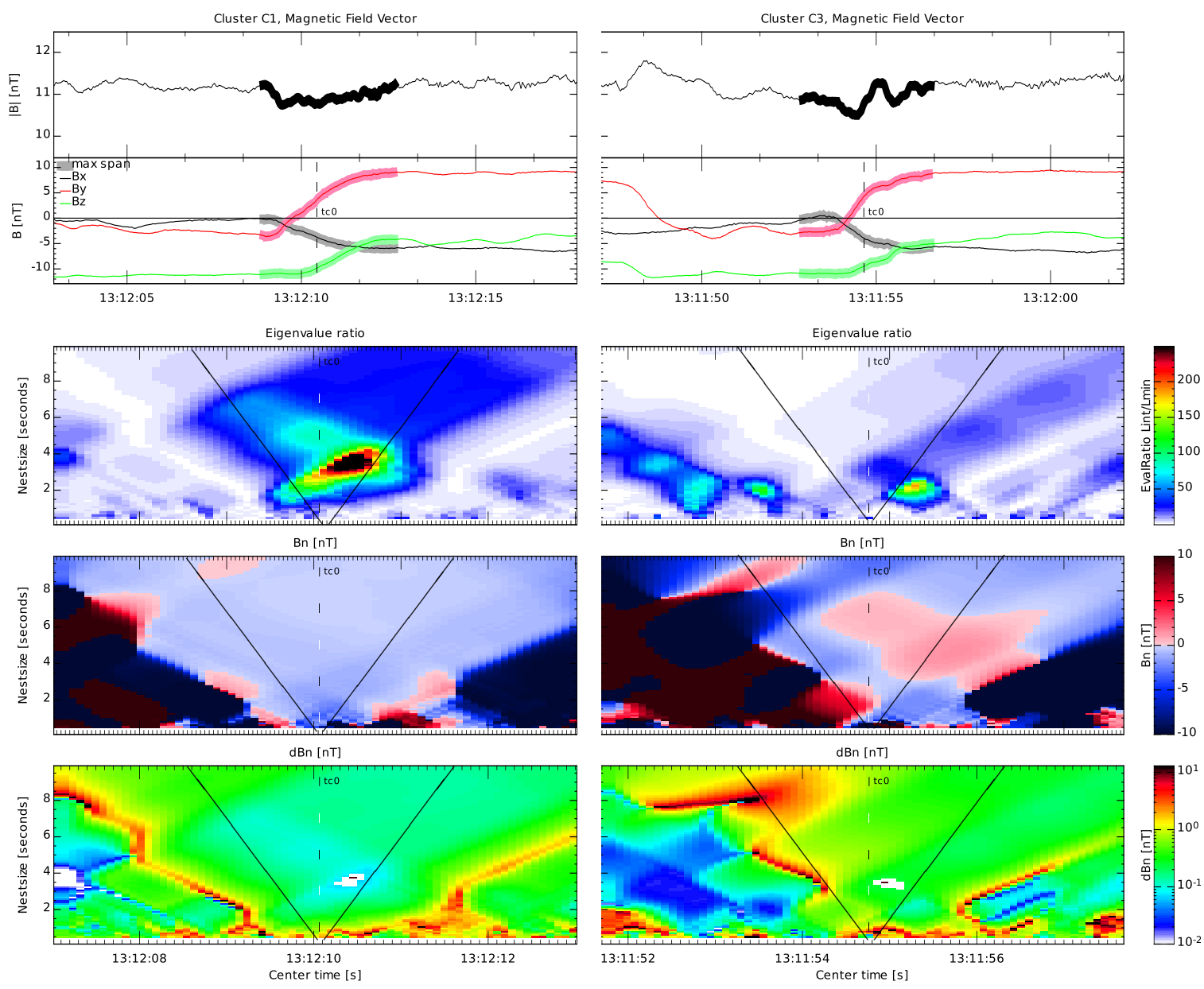

Fig. 2. Magnetic field time series and color-coded maps showing results from the MVAB analysis, for the 2003-018-1312 crossing (Event 1 in Tables 1 and 2) by $\mathrm{C} 1$ (left) and C3 (right). From top to bottom, the panels show B-field magnitude and components, thereafter maps of the eigenvalue ratio (EVR), the normal magnetic field $B_{n}$, and its statistical uncertainty $d B_{n}$. The color bar on the right applies to both C1 and C3. The white area in the bottom panels shows the location of the 20 nests with the lowest $d B_{n}$ within a 1/3-overlap cone that is indicated by the black lines (see text). The short black line within the white area marks the data interval with the lowest $d B_{n}$. The $B$-traces in the upper two panels for $\mathrm{C} 1$ and $\mathrm{C} 3$ are shown with thicker lines for the interval spanning the 20 nest members.

$$
\begin{aligned}
d B_{n} & =\left|\Delta\left\langle\boldsymbol{B} \cdot \boldsymbol{x}_{3}\right\rangle\right| \\
& =\sqrt{\frac{\lambda_{3}}{M-1}+\left(\Delta \varphi_{32}\langle\boldsymbol{B}\rangle \cdot \boldsymbol{x}_{2}\right)^{2}+\left(\Delta \varphi_{31}\langle\boldsymbol{B}\rangle \cdot \boldsymbol{x}_{1}\right)^{2}} .
\end{aligned}
$$

In these equations, $\lambda_{\mathrm{i}}$ and $\boldsymbol{x}_{\mathrm{i}}(i=1,2,3)$ are the eigenvalues and eigenvectors of the magnetic variance matrix, and $M$ is the number of data points in the nest being analyzed. We use the vector $\boldsymbol{x}_{3}$ as the estimator for the normal direction, where the corresponding $\lambda_{3}$ is the smallest eigenvalue. It represents the variance of the normal field component.

From the maps, one can now easily identify regions that satisfy certain requirements. It is obvious that not all combinations of center time and nest size shown in the figure make sense. We have therefore required that the analyzed time seg- ment should overlap with the initial center time $T_{\mathrm{c} 0}$ by at least $1 / 3$ of the respective data interval. Acceptable combinations of center time and data interval length then define a wedge in the maps, as illustrated by the black slanting lines in the map panels of Fig. 2.

After some experimentation, we found that the optimal boundary normal could be obtained by identifying the 20 nest members with the lowest $d B_{n}$ within the wedge that also have eigenvalue ratio $>20$. The location of these 20 nest members is shown as the white area in the bottom panels of Fig. 2. Comparison of the maps shows that the white area for $\mathrm{C} 1$ (on the left in the figure) is located where the EVR maximizes, and where $B_{n}$ is fairly uniform. The hodogram projections, for the time interval corresponding to the minimum $d B_{n}$ (the short black line within the white area) are shown in Fig. 3. For $\mathrm{C} 1$ (on the left in the figure), the almost 


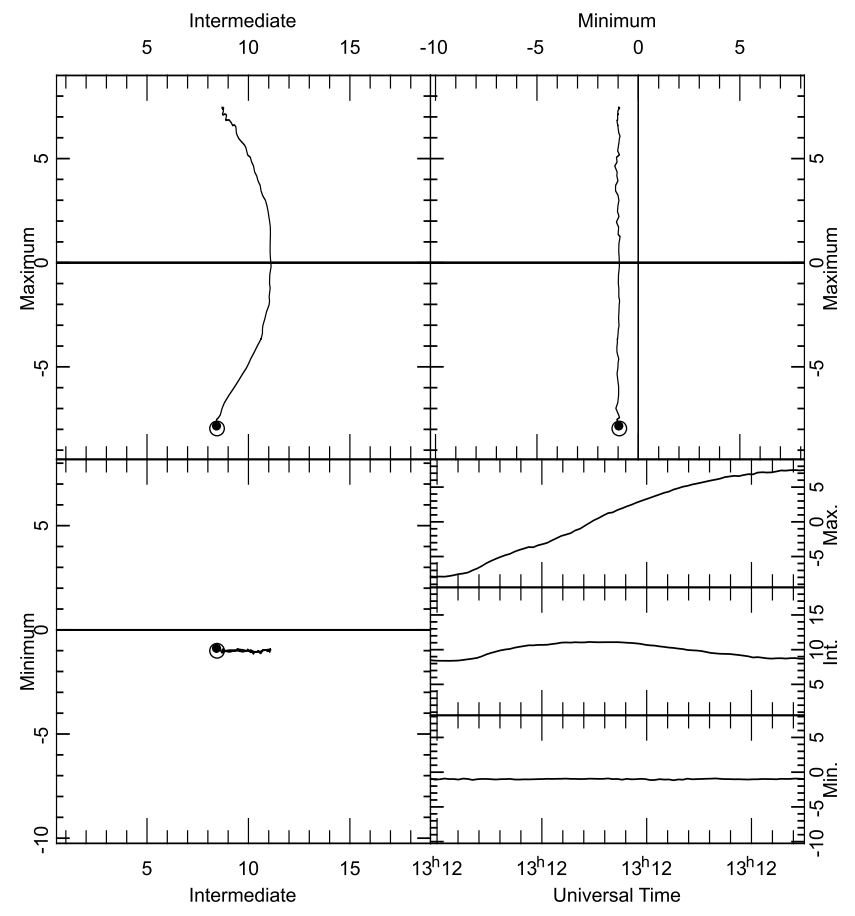

Time Interval (UT): 2003-01-18T13:12:08.937 - 13:12:12.505

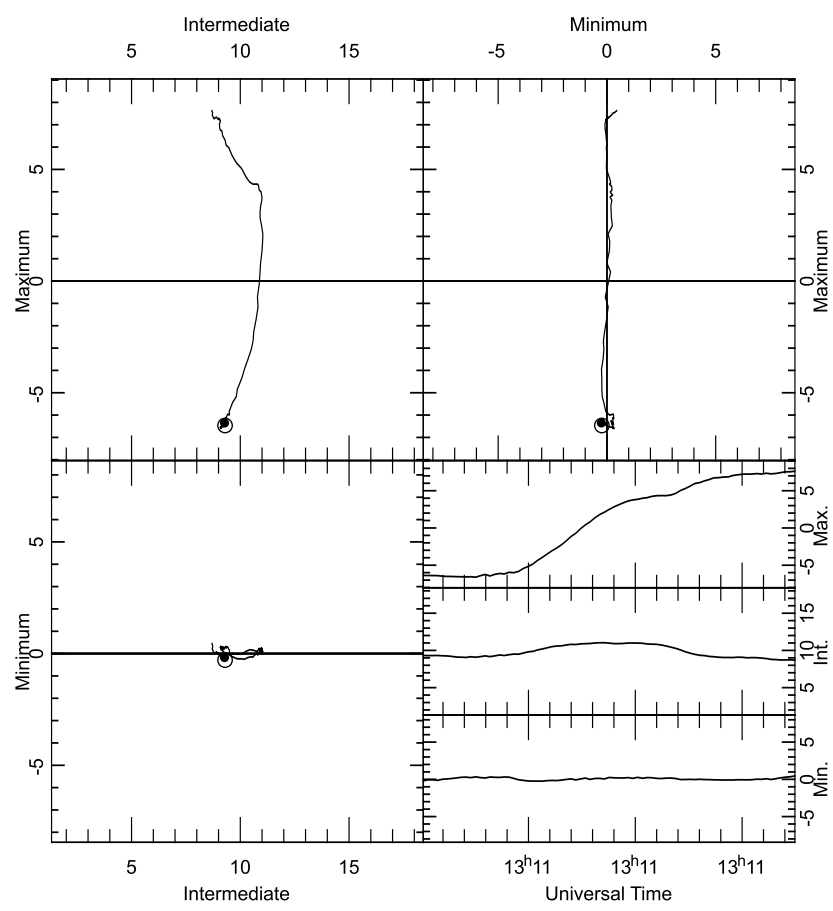

Time Interval (UT): 2003-01-18T13:11:53.015 - 13:11:56.494

Fig. 3. Top panel: $\mathrm{C} 1$ hodogram projections for the data interval with the lowest $d B_{n}$ in Fig. 2 (time span used is 13:12:08.9 to 13:12:12.6; eigenvalue ratio is 318). Bottom panel: corresponding hodograms for spacecraft C3 (time span used is 13:11:53:1 to 13:11:56.4; eigenvalue ratio is 27). Panels on the lower right show time series of the three components. All units are nT.

circular arc traced out by the tangential field (upper left projection) and the almost perfectly constant and non-zero normal component (upper right projection) are readily apparent. Note that the theoretical hodogram in Fig. 1 is rotated by $90^{\circ}$ relative to the observed tangential hodograms.

By contrast, the maps for the same event, recorded by $\mathrm{C} 3$ (right panel of Fig. 2), show that the white area not only is not co-located with the region of maximum EVR, but also straddles a $B_{n}$-sign boundary. The hodogram projections for the time interval corresponding to the minimum $d B_{n}$ (Fig. 3, right), confirm the uncertainty in the sign of $B_{n}$.

The differences between the results for $\mathrm{C} 1$ and $\mathrm{C} 3$, apparent in Fig. 2, and also between the hodograms (Fig. 3) highlight a common feature of the arc-polarized DDs seen by Cluster, namely significant differences in the nature of the transitions observed by the four spacecraft. These differences are apparent, not only in the maps, but already in the raw time series of the magnetic field, e.g., as displayed in the top panels of Fig. 2.

\subsubsection{Determination of $B_{n}$}

As our best estimate of $B_{n}$ we take the average of the $B_{n}$ values associated with the 20 nest members in the white areas in Fig. 2, and we take the standard deviation of these 20 values as our measure of the statistical uncertainty (this stan- dard deviation is typically much higher than the 20 individual $d B_{n}$ values in the white areas in Fig. 2). An additional error source is the uncertainty in the spin axis offset in the FGM measurements, estimated at no more than $0.2 \mathrm{nT}$ (A. Balogh, private communication). For a normal vector with a GSE Z-component $n_{\mathrm{z}}$, this implies an additional uncertainty of $0.2 n_{\mathrm{Z}} \mathrm{nT}$, which we include (by quadratic addition) in error estimates to be given later on.

\subsection{Determination of polarization}

The basic criterion for assigning the sense of polarization is as follows. Assume an observer rides on a plasma element as it crosses the discontinuity. If the sense of field rotation observed in that frame agrees with the ion sense of gyration in the normal field, $B_{n}$, then it is referred to as ion polarized; if the sense of field rotation agrees with that of an electron, it is electron polarized. Since the DD always blows past the observing S/C in the anti-solar direction, the sense of field rotation in a tangential hodogram, such as shown in Fig. 3, is the same as the sense seen by the above observer, only if the DD is propagating (relative to the plasma) away from the sun. If the DD propagates toward the sun instead, the sense of field rotation seen by the observer is opposite to that recorded in the hodogram. For a one-dimensional structure, the sense of propagation can be obtained by transforming the measured 
velocity data to the deHoffmann-Teller (HT) frame, and then checking the sign of $v_{n}=\left(v-\boldsymbol{V}_{\mathrm{HT}}\right) \cdot \boldsymbol{n}$, where $\boldsymbol{n}$ is the normal vector (pointing sunward, i.e., $n_{X}>0$ ) and $v_{n}$ is the normal speed of the plasma as seen in the DD rest frame. If $v_{n}>0$, propagation is away from the sun; if $v_{n}<0$, propagation is toward the sun.

From the above consideration follows that, if both $v_{n}$ and $B_{n}$ are positive, or if both are negative, then a clockwise tangential hodogram is ion polarized and a counter-clockwise hodogram is electron polarized. If $v_{n}$ is positive but $B_{n}$ is negative, or if $v_{n}$ is negative and $B_{n}$ is positive, then a clockwise hodogram corresponds to electron polarization and a counterclockwise one to ion polarization.

Instead of determining the actual value of $v_{n}$ we will make use of a basic property of the Walen relation to obtain the sign of $v_{n}$ relative to the sign of $B_{n}$. The Walén relation implies that, for an RD, the plasma velocity vector in the HTframe agrees either with the local Alfvén velocity vector or with its reverse, when $B_{n}$ and $v_{n}$ have the same sign or opposite signs, respectively. The slope of the regression line in a scatter plot of the components of ( $\left.\boldsymbol{v}-\boldsymbol{V}_{\mathrm{HT}}\right)$ against the corresponding components of $\boldsymbol{V}_{\mathrm{A}}$ is positive when $B_{n}$ and $v_{n}$ have the same sign, and negative when they have opposite signs. The above relationships can then be reduced to the following four combinations:

- Walén slope $>0$ and clockwise hodogram rotation: ion polarization

- Walén slope $>0$ and counter-clockwise hodogram rotation: electron polarization

- Walén slope $<0$ and clockwise hodogram rotation: electron polarization

- Walén slope $<0$ and counter-clockwise hodogram rotation: ion polarization

To obtain $\boldsymbol{V}_{\mathrm{HT}}$ and the Walén slopes, we used spin resolution ion plasma and magnetic field data for 1-min intervals centered on the DDs, based on the procedure described in Khrabrov and Sonnerup (1998b) (see also Sonnerup et al., 1987, and Paschmann and Sonnerup, 2008).

\subsection{Plasma $\beta$}

A quantity needed to evaluate the parameter $u_{2}$ (Eq. 4) is the plasma $\beta$, i.e., the ratio of the plasma (ion plus electron) and magnetic field pressures. The electron pressure we calculated from the temperatures, $T_{\|}$and $T_{\perp}$, and the density measured by the PEACE instrument (Johnstone et al., 1997) and provided by the CAA with adequate time resolution for spacecraft C2, albeit not for all our events. However, Cluster does not include a dedicated solar wind ion instrument and the CIS/HIA instrument, while providing sufficiently accurate densities and velocities in the solar wind, does not give accurate ion temperatures, as is apparent from comparisons with the ion temperatures measured by the SWE instrument on ACE. We therefore used the ACE temperatures, available from the CDAWeb, properly shifted to the times of the Cluster observations. The $\beta$ values thus obtained a range between 0.7 and 1.1, with electrons and ions contributing about equally.

\section{Results}

For all 26 cases meeting the pre-selection criteria, we ran the automated MVAB procedure and the Walén test described in Sects. 4.1 and 4.2. Tables 1 and 2 show the results.

From left to right, Table 1 lists the event number, Knetter's event ID; the cone angle $\Theta$ of Knetter's triangulation normal relative to the GSE X-axis; the sense of rotation of the tangential field in the hodogram; the Walén slope; the polarization determined from the sense of field rotation in combination with the Walén slope; the duration (in s) of the transition, determined from visual inspection of the magnetic field time series; the thickness, scaled to the ion inertial length, $\lambda_{\mathrm{i}}$, obtained by multiplying the durations with the DD-speeds and dividing the result by $\lambda_{\mathrm{i}}$, calculated from the plasma density; the shear angle of the magnetic field; Knetter's DD speed from triangulation; the plasma density; the magnetic field magnitude; and the deviation of the field strength, $B$, from its average, this deviation being expressed as the ratio $B_{\mathrm{dev}}=B_{\mathrm{z} 0} / B_{\mathrm{z} 2}$, where for simplicity the average of the field on the two sides of the DD is used as a proxy for $B_{\mathrm{z} 2}$. When a clear maximum or minimum in $B$ could be identified during the main field transition, this value was used for $B_{\mathrm{z} 0}$. In several events, notably the longer duration ones, no clear maximum or minimum could be identified, and a blank was entered in the the $B_{\operatorname{dev}}$ column.

Except for the triangulation results, the quantities in the table are taken from spacecraft $\mathrm{C} 1$ data, but most entries are the same or similar for the other three spacecraft. Events 4 and 10 are double-arc structures, which are listed as two separate single-arc events, labeled 4 and $4 s$ and 10 and $10 s$, the letter $s$ denoting the slow (electron) branch of the hodogram. Therefore, the total number of events in the table is 28 , rather than 26. Event 4 together with $4 s$ is the double-arc case discussed in Paper I.

Table 2 lists, for all four Cluster spacecraft, the cone angle between our optimal MVAB normals and Knetter's triangulation normal; the average EVR value; and the normal component of the magnetic field, the average $B_{n}$, together with its estimated $1 \sigma$ uncertainty. The average EVR, as well as $B_{n}$ and its uncertainty, is calculated from the 20 nest members in the white areas of the bottom maps in Fig. 2. In Table 2, an empty entry for a spacecraft indicates that the MVAB result did not meet the selection criterion $E V R \geq 20$. When none of the MVAB results from the four spacecraft had EVR $\geq 20$, the corresponding event was deleted from the table, leaving 22 events, of which only 10 had entries from all four 
Table 1. Arc-polarized structures: Overview of parameters.

\begin{tabular}{|c|c|c|c|c|c|c|c|c|c|c|c|c|}
\hline No & KnetterID & $\Theta$ & ArcRot & Wslope & Pol & Dur [s] & $\mathrm{d} / \lambda_{\mathrm{i}}$ & Angle & $V_{\text {cva }}$ & Dens & $B$ & $B_{\mathrm{dev}}$ \\
\hline 1 & 2003-018-131208 & 84.5 & counter & -1 & $\mathrm{i}$ & 3.5 & 16.6 & 79.9 & 231.9 & 21.5 & 11.5 & 0.96 \\
\hline 2 & 2003-022-224959 & 4.3 & clock & -0.81 & $\mathrm{e}$ & 10 & 63.0 & 72.4 & 673.7 & 4.5 & 8.8 & \\
\hline 3 & 2003-030-074630 & 42.0 & clock & 1.04 & $\mathrm{i}$ & 3 & 13.8 & 68 & 302.5 & 12 & 9.5 & 0.94 \\
\hline 4 & 2003-034-191133 & 18.8 & clock & 0.93 & $\mathrm{i}$ & 2.5 & 15.5 & 75.7 & 455.6 & 9.5 & 8.2 & 0.92 \\
\hline $4 s$ & 2003-034-191133s & - & counter & 0.93 & e & 300 & 1855.8 & 75.7 & 455.6 & 9.5 & 8.2 & \\
\hline 5 & 2003-037-082000 & 2.5 & clock & 0.87 & $\mathrm{i}$ & 3 & 17.4 & 75 & 481.2 & 7.5 & 7.4 & 0.87 \\
\hline 6 & 2003-037-083149 & 33.6 & counter & 0.96 & $\mathrm{e}$ & 3 & 15.1 & 71.7 & 404.0 & 8 & 7.4 & 1.07 \\
\hline 7 & 2003-037-101832 & 30.1 & counter & 0.78 & $\mathrm{e}$ & 1.5 & 6.1 & 56.9 & 327.8 & 8 & 7.2 & 1.1 \\
\hline 8 & 2003-041-144722 & 43.7 & clock & 0.82 & $\mathrm{i}$ & 4 & 12.6 & 103.1 & 292.4 & 6 & 7 & 0.96 \\
\hline 9 & 2003-042-021103 & 52.9 & clock & 0.82 & $\mathrm{i}$ & 3 & 9.3 & 69.7 & 256.5 & 7.5 & 6.7 & 0.94 \\
\hline 10 & 2003-046-160842 & 17.4 & counter & -0.84 & i & 0.5 & 1.5 & 57.3 & 335.6 & 4.3 & 8.4 & 0.88 \\
\hline $10 s$ & $2003-046-160842 \mathrm{~s}$ & & clock & -0.84 & $\mathrm{e}$ & 19 & 58.2 & 57.3 & 335.6 & 4.3 & 8.4 & \\
\hline 11 & 2003-048-191709 & 63.1 & clock & -0.82 & $\mathrm{e}$ & 10 & 23.0 & 87.2 & 270.9 & 3.7 & 6.5 & \\
\hline 12 & 2003-048-234901 & 28.1 & clock & -0.89 & $\mathrm{e}$ & 3 & 21.4 & 69.5 & 610.9 & 7 & 14.2 & \\
\hline 13 & 2003-063-105758 & 32.0 & clock & 0.91 & $\mathrm{i}$ & 2.5 & 11.3 & 70.1 & 482.5 & 4.5 & 9.2 & 0.93 \\
\hline 14 & 2003-063-120223 & 30.0 & clock & 0.84 & $\mathrm{i}$ & 3 & 13.3 & 88 & 448.9 & 5 & 8.1 & 0.9 \\
\hline 15 & 2003-063-121338 & 56.8 & clock & 0.94 & $\mathrm{i}$ & 6 & 12.5 & 103.6 & 210.7 & 5 & 7.4 & 0.91 \\
\hline 16 & 2003-070-013700 & 17.3 & clock & 0.78 & $\mathrm{i}$ & 1.5 & 8.1 & 65.6 & 377.5 & 10.5 & 7.2 & 0.91 \\
\hline 17 & 2003-070-035310 & 36.9 & clock & 0.83 & $\mathrm{i}$ & 1.5 & 7.1 & 65 & 347.7 & 9.5 & 7.2 & 0.9 \\
\hline 18 & 2003-070-042755 & 7.1 & counter & 0.71 & e & 2.5 & 13.4 & 70.1 & 394.7 & 9.5 & 7.4 & \\
\hline 19 & 2003-070-094213 & 28.5 & counter & 0.78 & $\mathrm{e}$ & 13.5 & 67.0 & 78.7 & 339.8 & 11 & 7.6 & \\
\hline 20 & 2003-070-094508 & 32.4 & clock & 0.84 & $\mathrm{i}$ & 5 & 25.8 & 88.4 & 352.8 & 11 & 7.6 & 0.9 \\
\hline 21 & 2003-082-074824 & 13.9 & counter & -0.85 & $\mathrm{i}$ & 2 & 10.4 & 78.1 & 639.4 & 3.4 & 6.6 & 0.92 \\
\hline 22 & 2003-082-080921 & 18.4 & clock & -0.74 & e & 1 & 4.7 & 69.4 & 588.9 & 3.3 & 6.2 & 1.17 \\
\hline 23 & 2003-084-134355 & 54.2 & counter & -0.7 & $\mathrm{i}$ & 6 & 14.7 & 73.8 & 210.9 & 7 & 4 & 0.93 \\
\hline 24 & 2003-084-135511 & 21.1 & counter & -0.72 & $\mathrm{i}$ & 15 & 67.6 & 68.9 & 386.4 & 7 & 3.9 & \\
\hline 25 & 2003-091-215425 & 40.5 & clock & 0.74 & $\mathrm{i}$ & 2.5 & 9.8 & 81.1 & 398.2 & 5 & 4.8 & 0.91 \\
\hline 26 & 2003-091-224013 & 15.2 & counter & 0.9 & $\mathrm{e}$ & 18 & 85.6 & 70.8 & 520.8 & 4.3 & 5 & \\
\hline
\end{tabular}

spacecraft. In the table, the total number of individual spacecraft crossings satisfying EVR $\geq 20$ is 65 .

A set of histograms is shown in Fig. 4 to illustrate the results and some general properties of the DDs in our data set. In particular, histograms of the distribution of $\boldsymbol{B}_{n}, \mathrm{EVR}$, and cone angle (between the triangulation normal and the MVAB normal) from Table 2 are shown in parts (e), (f), and (g) of the figure. For these three histograms, only crossings with eigenvalue ratios $\geq 20$ are included. We now proceed to discuss the DD properties in detail.

\subsection{General properties}

The magnetic shear angles for our events range from $57^{\circ}$ to $104^{\circ}$ (see Fig. 4a). Plasma densities and magnetic field strengths have typical values, ranging between 3 and $22 \mathrm{~cm}^{-3}$, and between 4 and $14 \mathrm{nT}$, respectively. The events occurred under both slow and fast solar wind conditions, as the histogram in Fig. 4d illustrates.

According to Table 1, the duration of the crossings ranges from $0.5 \mathrm{~s}$ to $300 \mathrm{~s}$, with the majority below $5 \mathrm{~s}$. Only 7 have durations $\geq 10 \mathrm{~s}$. This means that, in the plasma moments with their $4 \mathrm{~s}$ resolution, most of the crossings are unresolved. For the histogram of the durations in Fig. 4b, the event with $300 \mathrm{~s}$ duration was not included. As shown in the table, the thicknesses scaled to $\lambda_{\mathrm{i}}$ range between 1.5 and 1856, with the majority below 30, as the histogram in Fig. 4c illustrates. For comparison, Lepping and Behannon (1986), using Mariner 10 magnetic field measurements at distances in the range $0.46-1 \mathrm{AU}$, reported thicknesses of $36 \pm 5$ local proton gyro radii for both TDs and RDs. They also noted that thicknesses were smaller closer to Earth. Vasquez et al. (2007), using high resolution magnetic field from the ACE spacecraft (near $1 \mathrm{AU}$ ), reported thicknesses in the range 4-8 ion gyro radii. These results are qualitatively consistent with those reported here.

As shown in Sect. 2 (see Eq. 7), the magnitude of the quantity $\left(d / \lambda_{\mathrm{i}} \cdot\left(B_{\operatorname{dev}}-1\right)\right)$ should increase with the magnetic shear angle. Figure 5 illustrates that such a relationship exists, albeit with a lot of scatter. One reason for this scatter is that, in our data analysis, neither $\boldsymbol{B}_{\operatorname{dev}}$ nor the thickness $d$ was defined in a precise manner. More importantly, the individual $\beta_{2}$ values, $\gamma$ values, and $\theta_{2}$ values, all of which enter into the expression for $u_{2}$ (see Eq. 4), are not taken into account in the figure. Additionally, modulation of the thickness, resulting from the presence of structures such as magnetic flux ropes embedded within the DDs, would contribute to the scatter. 
Table 2. Arc-polarized structures: four-spacecraft results.

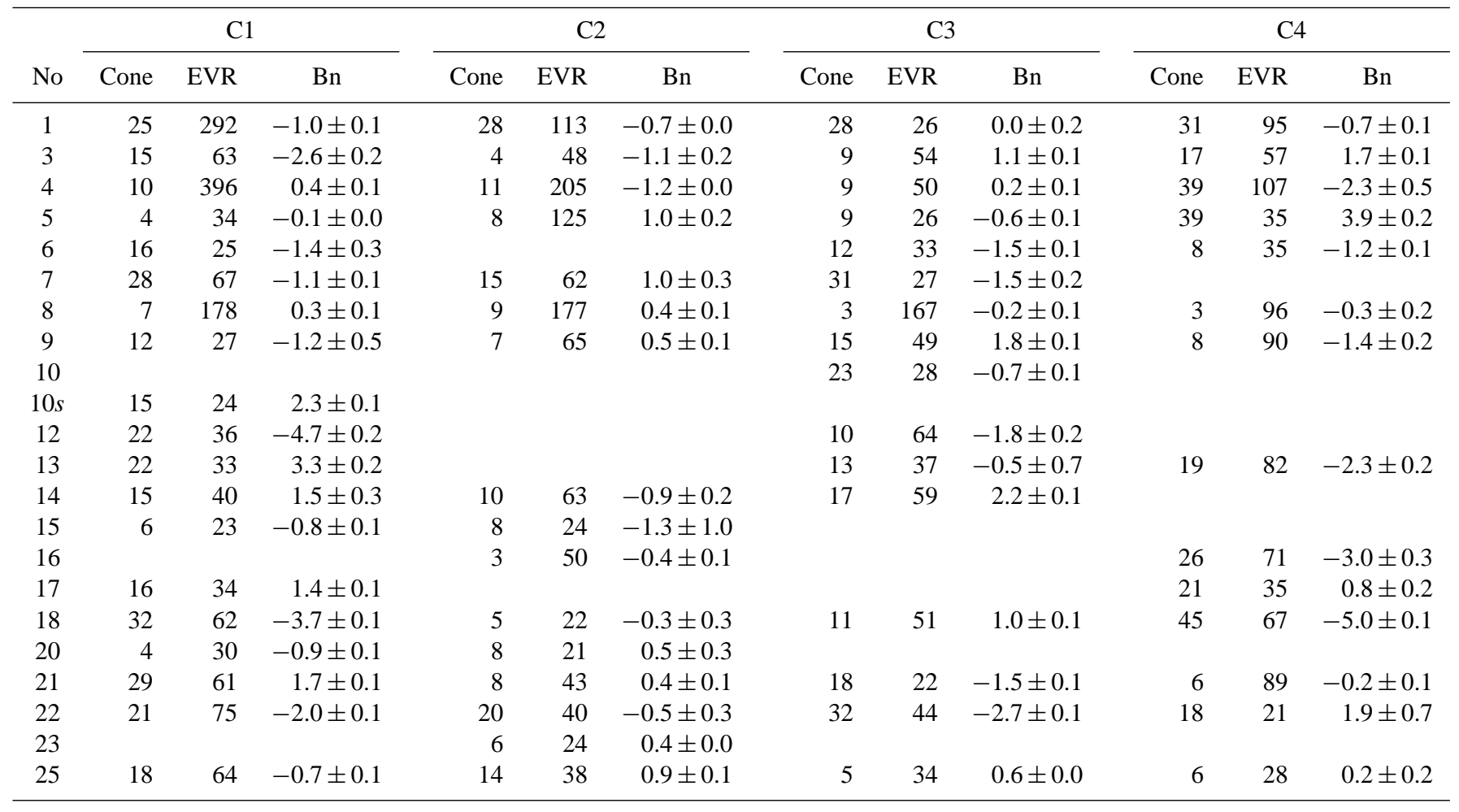

As mentioned in Sect. 4.3, the sample $\beta$ values we calculated, ranging between 0.7 and 1.1. The $\theta_{2}$ value that enters into $u_{2}$ also varies from event to event but is usually close to $90^{\circ}$. The $\gamma$ values in the expression for $u_{2}$ can presumably also vary significantly from event to event. From Eq. (7) it is seen that, if all events had the same value of $u_{2}$, the relationship shown in Fig. 5 should be represented by a straight line through the origin of slope proportional to $u_{2}$, with negative slope for ion-polarized DDs (shown in red), and equal but positive slope for electron-polarized DDs (of which there are only three cases, shown in blue in the figure). The corresponding solid lines shown in the figure represent the best fit to the data of a straight line through the origin. The slope magnitudes of these lines indicate that, on average, the $u_{2}$ value was 0.49 for the ion-polarized events and 0.46 for the electron-polarized ones. Given the uncertainties evident in the plot, there is no statistically significant difference between these two values, a result that is consistent with our theory. With an assumed average $\beta_{2}$ value of 0.8 (and putting $\theta_{2}=90^{\circ}$ ), we then find an average $\gamma$ value of 1.24 and 1.16 for the ion- and electron-polarized branches, respectively. This result is qualitatively consistent with the conclusion in Paper I that the effective $\gamma$ is close to its isothermal value. As mentioned there, the physical explanation for this behavior is not clear.

\subsection{MVAB results: eigenvalue ratios, normals, and nor- mal components}

Even though our initial pre-selection only required Knetter's routine MVAB to give EVR $>5$, our MVAB procedure gave EVR $>20$ for 65 individual crossings out of the total of 112 such crossings for the 28 events in Table 1. This fact illustrates the benefit of our automated two-dimensional search for an optimal data interval for MVAB. However, for the long duration events (events 2, 4s, 10s, 11, 19, 24, and 26 in Table 1), the automated analysis gave too short data segments and was replaced by running MVAB manually for longer time intervals. Except for event 10s, those results do not appear in Table 2, because they all gave EVR $\leq 20$.

Table 2 and Fig. 4e show that most $B_{n}$ values are small: 53 of the 65 values, or $82 \%$, are $\leq 2 \mathrm{nT}$. The magnitude of $B_{n}$ exceeds the $1 \sigma$ error estimate given in Table 2 in all but 4 individual crossings: at the $2 \sigma$ level in all but 11 cases and at $3 \sigma$ in all but 14 cases.

Even using this latter most conservative error estimate, there are 51 individual spacecraft crossings that appear to have a significant non-zero normal field component. A few of these cases (e.g., case 12 for $\mathrm{C} 1$; case 5 for $\mathrm{C} 4$; case 18 for $\mathrm{C} 4$ ) have substantial values of $B_{n}$ and some of those have large EVR values as well. All the same, one must ask if some of these exceptional cases could be the result of significant 2 D or 3-D embedded structures, such as magnetic flux ropes. 

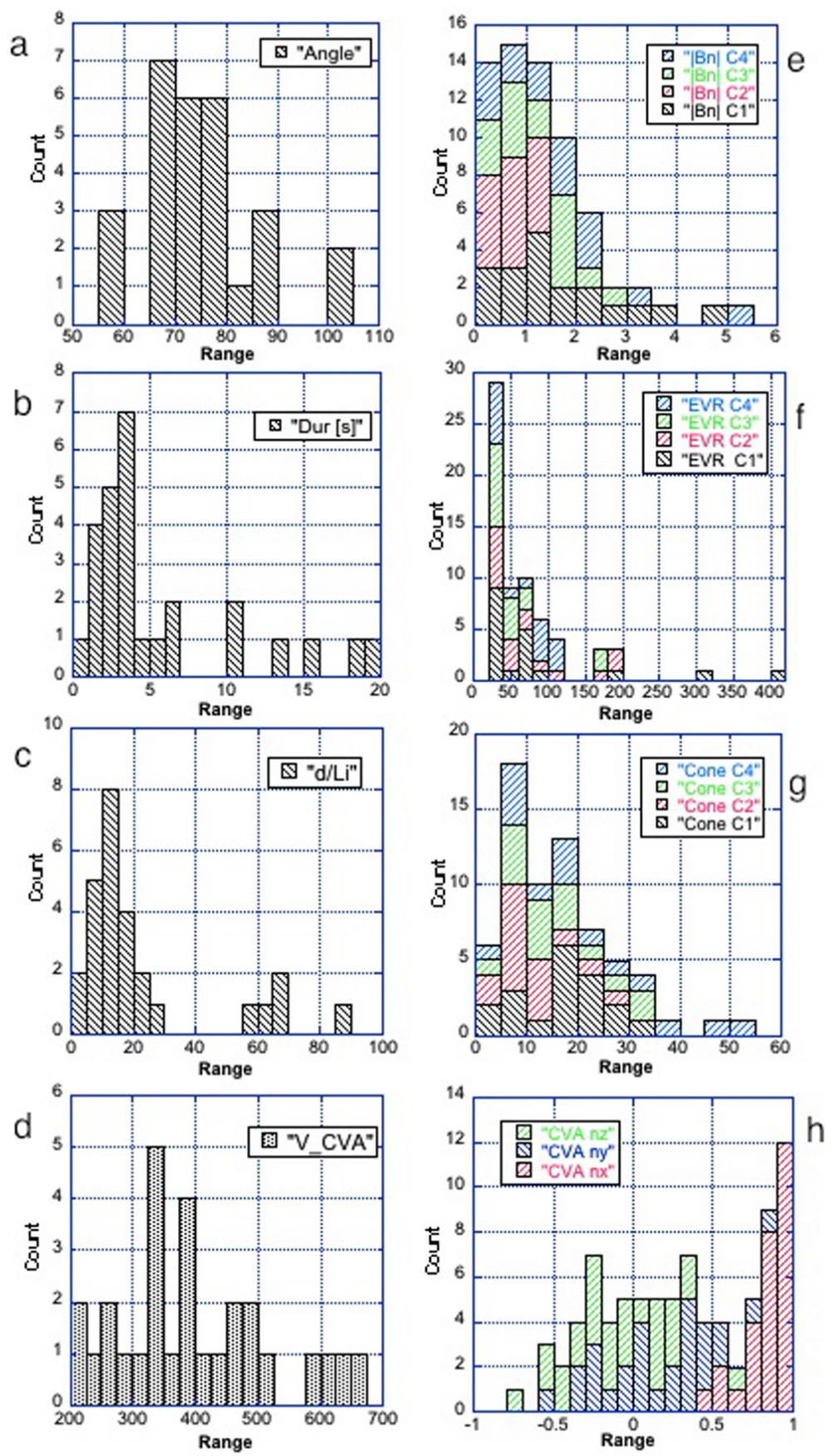

Fig. 4. Histograms of key quantities. Left, top to bottom: (a) field shear angle across DD; (b) DD-duration; (c) DD-thickness in units of the ion inertial length $\lambda_{\mathrm{i}}$ (labeled d/Li in figure); (d) DD-speeds from triangulation. Right: (e) $B_{n}$ values for C1-C4 in standard Cluster color coding (black: $\mathrm{C} 1$, red: $\mathrm{C} 2$, green: $\mathrm{C} 3$, and blue: $\mathrm{C} 4$ ); (f) associated eigenvalue ratios; (g) cone angles between MVA and triangulation normals for $\mathrm{C} 1-\mathrm{C} 4$; (h) components of the triangulation normals themselves. For the $B_{n}$, eigenvalue ratio, and cone angle histograms, all cases with eigenvalue ratios $\leq 20$ were excluded. For Event $4 s$, the duration and thickness are off-scale.

The reconstruction results by Teh et al. (2011) indicate that such structures can cause the minimum variance direction to be a poor predictor of the DD orientation and of $B_{n}$ and can cause the cone angle of the MVAB normal relative to the triangulation normal to be large.
Table 2 shows that, for a given event, the $B_{n}$ values observed by the various Cluster spacecraft can be quite different, including differences in sign. This is the situation already observed and discussed in Paper I, where the normals obtained for the four spacecraft differed significantly 


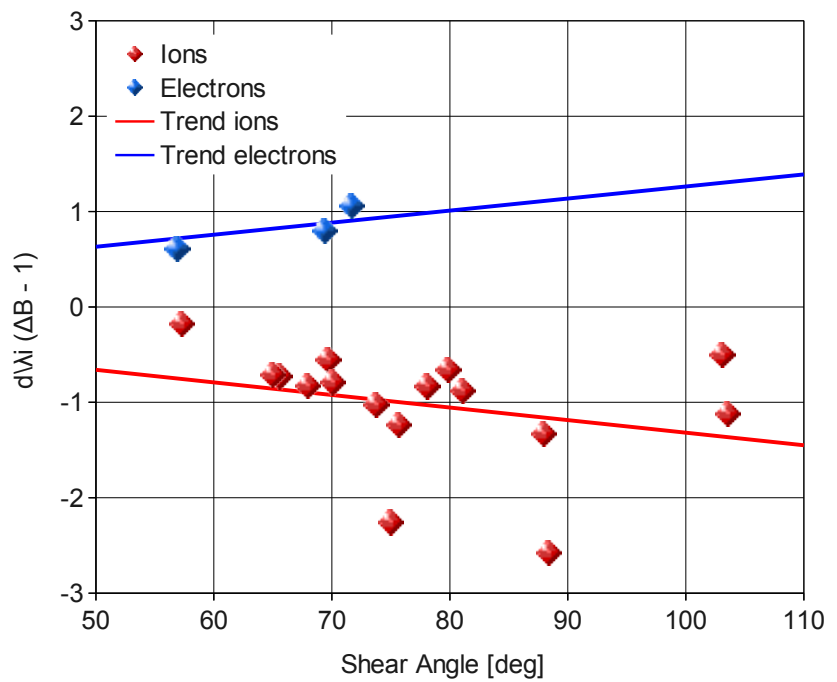

Fig. 5. Field deviation, in the form $\left(d / \lambda_{\mathrm{i}}\right)\left(B_{\mathrm{dev}}-1\right)$, versus the field shear angle, based on the values in Table 1. Electron-polarized cases (three points only) are shown in blue and ion-polarized cases in red. Best-fit straight lines through the origin (off-scale) are shown as solid lines. The plot is based on data from Cluster 1.

among themselves and from the triangulation normal. For the present cases, Table 2 and Fig. $4 \mathrm{~g}$ show the deviations from the timing normals expressed as the cone angles between the MVAB and triangulation normals. The great majority (91\%) of these angles are less than $30^{\circ}$, and $71 \%$ are less than $20^{\circ}$. But even $20^{\circ}$ is usually much too large an angle to permit a meaningful determination, by triangulation, of the small normal field components associated with most of the discontinuities. One is forced to conclude that the triangulation normals are not useful for this purpose.

The angle between individual normal vectors for the events in Table 2 ranges up to $73^{\circ}$ with an average of $20^{\circ}$. The spread in normal directions among the four spacecraft is illustrated and discussed in Paper I (Fig. 6) for events $4 / 4 s$. That such large differences in orientation occur for Cluster spacecraft separations of only a few thousand $\mathrm{km}$ was considered improbable in the past (e.g., Neugebauer, 2006). These differences indicate that DDs often possess local deformations and/or internal structure on scales smaller than the spacecraft separation, even if the large-scale orientation of the DD is well defined.

The eigenvalue ratios, associated with those $B_{n}$ values and cone angles, shown in Fig. 4f, are all $\geq 20$ by definition, and $58 \%$ are $\geq 40$. Even though exceptions may exist, such large EVRs tend to lend credence to the $B_{n}$ values obtained. The MVAB normal vectors we obtained are distributed as shown in Fig. 4h. This figure shows the dominance of normals with large GSE X-components, a result imposed by the exclusion of cases with bow shock connection.

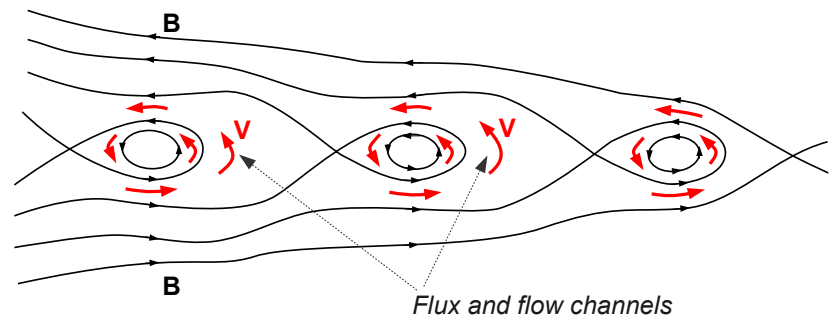

Fig. 6. Schematic drawing for positive Walén slope, showing channels of inter-connected field and flow, located between magnetic islands and also field-aligned flow within the islands. Note that on the left side of each island the normal field and flow both point downward, while on the right side they both point upward. The flow along the intervening narrow channels that magnetically connect the two sides of the directional discontinuity (DD) is upward in the figure, a situation that corresponds to downward propagation relative to the plasma. However, unless it can be established that the observing spacecraft was in fact located in such a channel during its crossing of the structured DD, the overall direction of propagation of the DD cannot be established, even if $B_{n}$ and $v_{n}$ are well determined.

\subsection{Sense of propagation}

For a discontinuity having purely one-dimensional structure, the criterion for determining the propagation sense is as follows: If $B_{n}$ and the Walén slope have the same sign, the DD propagates away from the sun; if they have opposite signs, it propagates toward the sun. From Tables 1 and 2, one can then deduce that, according to this criterion, there would be 27 individual crossings showing clear $\left(\left|B_{n}\right|>3 \sigma\right)$ antisunward propagation and 24 crossings showing clear sunward propagation. In fact, for no single event do all four spacecraft unambiguously indicate the same propagation sense. This unexpected and unexplained behavior was encountered already in Paper I. Here we note that a DD model containing embedded islands of length comparable to the spacecraft separation could automatically account for this behavior. Plasma flowing along (or opposite to) the magnetic field within an island would automatically reverse its normal component, as the field reverses its normal component, from one end of an island to the other. And this behavior would not have anything to do with sunward or antisunward propagation. To account for the near constancy of the field and the approximately Alfvénic flow, one would have to assume that the islands developed as a result of growing tearing modes, perhaps with different reconnection rates at the various magnetic $\mathrm{X}$-points, in a propagating rotational discontinuity in which the normal field was weak. In such a scenario, there would be channels, located between islands, where actual magnetic connection and plasma flow, from one side of the DD to the other, would be present, as illustrated schematically in Fig. 6 . But, unless we could determine that one of the spacecraft was actually located in such a channel, we would not be able to 
draw any conclusion from our data about the actual propagation sense relative to the plasma.

\subsection{Polarization, thickness, and field magnitude}

As described in Sect. 4.2, the polarization was obtained from magnetic field hodograms, such as shown in Fig. 3, together with the sign of the Walén slope. According to Table 1, 17 of the total of 28 cases are ion polarized and 11 are electron polarized. Table 1 is based on data from $\mathrm{C} 1$, but the results from C3 are the same. Since CIS/HIA data are not available for C2 and $\mathrm{C} 4$, the polarizations for those spacecraft cannot be directly established, but we expect they would be the same.

Of the 11 electron-polarized events, 6 have durations $\geq 10 \mathrm{~s}$, while only one ion-polarized case has a duration $\geq 10$ s. In terms of scaled thicknesses, 7 of the 9 cases with $d / \lambda_{\mathrm{i}}>20$ are electron cases (as before, $\lambda_{\mathrm{i}}$ is the ion inertial length). Thus, electron-polarized events commonly have long durations, while ion-polarized cases rarely do.

In only 3 of the 11 electron-polarized cases could a clear maximum in $B$ be identified (these are the cases included in Fig. 5), while for the rest the situation is not clear, as a result of large variability. Of the 17 ion-polarized cases, all but one have a clear minimum in $B$. This minimum is apparent in the examples shown in the top panels of Fig. 2. The exception is event 24, where no clear minimum could be identified. Minima in $B$ for ion-polarized events and maxima for electronpolarized events are key predictions of the theory (see Eq. 2). Overall, this prediction is borne out in our study.

\section{Summary and conclusions}

Our results are summarized in the following items:

- Great care and caution are needed in the determination of the magnetic field component normal to the discontinuity layer. This component is usually very small and our experience is that, if it does not come out small, there is good reason to ask if the normal vector used is reasonable. Using the triangulation method, under the assumptions of a constant velocity of the discontinuity and an unchanging orientation, with data from the four Cluster spacecraft, it has generally been found (Knetter et al., 2004; Knetter, 2005) that the uncertainties in the normal direction from the timing method are so large that the resulting normal field component does not differ significantly from zero. The methodology we have developed is based on minimum variance analysis of the magnetic field (MVAB), applied to a series of different data intervals that are shifted relative to each other, both in terms of center time and duration, in a search for a minimum in the estimate of the statistical error of the normal field component. It usually gives small normal field components, which in many cases are nevertheless statistically different from zero. However, even this ex- tensive search for the optimal data intervals for MVAB occasionally can give completely erroneous results, for example, as a result of the presence of pronounced 2-D and 3-D structures within the DD (Teh et al., 2011).

- The normal field component and normal direction often come out significantly different for the four spacecraft. We have concluded that such inconsistencies represent real effects associated with local structure and/or time dependence. On the other hand, the sign of the Walén slope, which we could only determine for $\mathrm{C} 1$ and $\mathrm{C} 3$, is always the same. For the Cluster event discussed in $\mathrm{Pa}-$ per I, data from the PEACE instrument have been used (after publication of the paper) to show that there is in fact sign agreement of the Walén slopes between all four spacecraft.

- From the sign of the Walén slope, together with the sign of the normal field component, the propagation direction (relative to the plasma) of a one-dimensional discontinuity away from, or toward, the sun can in principle be determined. When determined according to this rule, the overall distribution of sunward and antisunward propagation in our data set is about 50-50, a result that is in disagreement with reports in the literature (e.g., Neugebauer, 2004) to the effect that propagation is usually away from the sun. In none of our events is the propagation sense, determined in this manner, unambiguously the same for all four spacecraft. We argue that such behavior is not an artifact but is associated with actual local spatio-temporal effects, which can degrade, or completely eliminate, our ability to determine the propagation sense (see Fig. 6).

- As predicted by the theory provided in Paper I (and expanded in Sect. 2 of the present paper), discontinuities having the ion sense of polarization display a slightly reduced magnitude of the magnetic field in their interior, while discontinuities having the electron polarization tend to have a weak field maximum instead. However, for the electron-polarized discontinuities, the effect is usually more difficult to establish, partly because they are almost always of much longer duration than the ion ones, and, as a result, contain field fluctuations that tend to obscure the effect.

- Arc-polarized structures should have the following three, readily observable, and robust features: Sign of Walén slope; sense of field rotation in the tangential hodogram projection; and presence of a field maximum or minimum in the middle of the discontinuity. According to the theoretical model developed in Paper I, these features are not all independent. They can therefore be used to provide a consistency check on the applicability of the theory. Specifically, the presence of a field maximum (minimum) should indicate the electron (ion) 
sense of polarization. The observed sense of field rotation in the hodogram, together with the sign of the Walén slope, will also tell the sense of polarization, and the two methods should give the same result. In all the 19 events in Table 1, in which a clear field maximum or minimum was present in the $\mathrm{C} 1$ data, the two results are indeed the same. This fact provides persuasive evidence that the qualitative aspects of the theory are consistent with the observations.

- Section 2, and also Appendix A, of our paper contains simple formulas that allow one to estimate the size of the field magnitude variation in ion- and electronpolarized discontinuities as well as their thickness. For our real events, the various quantities in the formulas are not sufficiently well determined to allow for precise detailed tests of the theory. However, in a qualitative sense, the Hall MHD theory appears to correctly describe the observed behavior. In particular, it predicts that arcpolarized structures can have thicknesses of several tens of ion inertial lengths. Mostly, the electron-polarized events are significantly thicker than the ion events, a feature not accounted for by the theory. Another unexplained fact is that the magnitude of the Walén slopes, which according to the theory should be extremely close to one, in fact often falls below one. The presence of alpha particles and pressure anisotropy (with $p_{\|}>p_{\perp}$ ) can provide at least a partial explanation. A final unexplained feature is the near-isothermal behavior required for the theory to adequately account for the magnitude of the field magnitude variations seen in the DDs.

- A new concept, developed in Paper I and pursued further in the present paper, is that the arc-polarized DDs in the solar wind represent a transition from one turning point to the next in the infinite non-linear and nondissipative wave solution given in Paper I. These turning points, at which the plasma flow along the normal direction is exactly equal to the Alfvén speed based on $B_{\mathrm{x}}$, are not true fixed points of the system. But the rate of change of the field vector is extremely slow there, so that minor effects not described by the theory, e.g., slow time evolution of the discontinuity thickness, or deviations from pressure isotropy, can probably allow the turning points to, in effect, serve as fixed points of the equations. This concept is very different from one that forms the basis of the $\mathrm{Wu}$ and Lee (2000) paper, in which intermediate shocks (by necessity dissipative and always leading to a field rotation angle of precisely $180^{\circ}$ ) are assumed to play a role comparable to that of rotational discontinuities. As discussed in Appendix B, our new concept can also be used to describe dissipation-free rotational field structures, such that the field behavior significantly violates our selection criterion of near constancy of the field magnitude. The theory predicts that such structures can be much thinner than most of the arc-polarized, solar wind structures discussed here, down to a few ion inertial lengths (see Fig. B2). However, our 1-D theory has difficulty accounting for very small values, such as $d / \lambda_{i 2}=1.5$ observed for Event 10 (see Table 1). One possible explanation for such a small width may be the presence of 2-D/3-D structures embedded within the DD, such as magnetic islands separated by regions near magnetic $\mathrm{X}$-points where the DD is much thinner.

\section{Appendix A}

In Paper I, the procedure is described for obtaining exact nonlinear wave solutions, in parametric form, of the ideal HallMHD equations, as exemplified in Fig. 1. For sufficiently small values (at the fixed Point 2) of $\epsilon_{2}^{2} \equiv\left(A_{\mathrm{x} 2}^{2}-1\right)$, the solution, evaluated at the top of the two hodogram branches (these locations, where $B_{\mathrm{y}}=0$, are denoted by the subscript 0 ), can be obtained in a simple form by power series expansion in the quantity $\epsilon_{2}$, in which one writes $A_{\mathrm{x} 0}^{2}-1=$ $a \epsilon_{2}+b \epsilon_{2}^{2}+O\left(\epsilon_{2}^{3}\right)$ and $B_{\mathrm{z} 0} / B_{\mathrm{z} 2}-1=c \epsilon_{2}+d \epsilon_{2}^{2}+O\left(\epsilon_{2}^{3}\right)$. Here the quantities $a, b, c$, and $d$ are coefficients that can be determined by substitution into the original equations in Paper I. After straightforward but lengthy algebra, the resulting expressions are

$$
\begin{aligned}
A_{\mathrm{x} 0}^{2}= & 1 \pm \epsilon_{2} \sqrt{\left(4 / u_{2}\right) \sin ^{2}\left(\Psi^{*} / 2\right)+\epsilon_{2}^{2}} \\
& +\epsilon_{2}^{2}\left[1+\frac{2 \gamma(\gamma+1) \beta_{2} \sin ^{2}\left(\Psi^{*} / 2\right)}{3 u_{2}^{2} \sin ^{2} \theta_{2}}\right], \\
\frac{B_{\mathrm{z} 0}}{B_{\mathrm{z} 2}} & 1 \pm \epsilon_{2} \sqrt{4 u_{2} \sin ^{2}\left(\Psi^{*} / 2\right)+u_{2}^{2} \epsilon_{2}^{2}} \\
& +\epsilon_{2}^{2}\left(2 \sin ^{2}\left(\Psi^{*} / 2\right)\right) \\
& \cdot\left[-2 \gamma-u_{2}+\frac{(\gamma+1)\left(\gamma \beta_{2}-3 \cos ^{2} \theta_{2}\right)}{3 u_{2} \sin ^{2} \theta_{2}}\right]
\end{aligned}
$$

where the + sign applies to the upper (electron-polarized) hodogram branch and the - sign to the lower (ion-polarized) branch. In both equations, terms of $O\left(\epsilon_{2}^{3}\right)$ have been neglected. As long as $\sin ^{2}\left(\Psi^{*} / 2\right) \gg \epsilon_{2}^{2}$, the terms $\epsilon_{2}^{2}$ and $u_{2} \epsilon_{2}^{2}$ under the two square root signs are $O\left(\epsilon_{2}^{3}\right)$ and can be ignored. In fact, these terms do not result from the expansion procedure. They were added in order to include the behavior when $\sin ^{2}\left(\Psi^{*} / 2\right)=0$. In that case, the two turning points coincide and should be located on the lower branch where $A_{\mathrm{x} 0}^{2}=1$ and (as can be shown) $B_{\mathrm{z} 0} / B_{\mathrm{z} 2}=\left(1-u_{2} \epsilon_{2}^{2}\right)$, behavior that is now obtained from Eq. (A1) and (A2). Note also that, when these terms are neglected, the terms proportional to $\epsilon_{2}$ are those given in Eqs. (1) and (2).

The accuracy of Eqs. (A1) and (A2) can be tested by use of results from the exact equations for the blue curve in Fig. 1, for which the following parameter values apply: $\Psi^{*}=\pi / 2$, 
Table A1. Quantitative comparison.

\begin{tabular}{lccccc}
\hline & \multicolumn{2}{c}{ Top of upper (electron) branch } & & \multicolumn{2}{c}{ Top of lower (ion) branch } \\
\cline { 2 - 3 } \cline { 5 - 6 } Quantity & $A_{\mathrm{x} 0}^{2}$ & $B_{\mathrm{z} 0} / B_{\mathrm{z} 2}$ & & $A_{\mathrm{x} 0}^{2}$ & $B_{\mathrm{z} 0} / B_{\mathrm{z} 2}$ \\
\hline From Paper I & 1.0477398 & 1.04181769 & & 0.9582725 & 0.95217432 \\
From Eqs. (A1) and (A2) & 1.047729 & 1.041717 & & 0.958287 & 0.952275 \\
From Eqs. (1) and (2) & 1.044721 & 1.044721 & & 0.955279 & 0.955279 \\
\hline
\end{tabular}

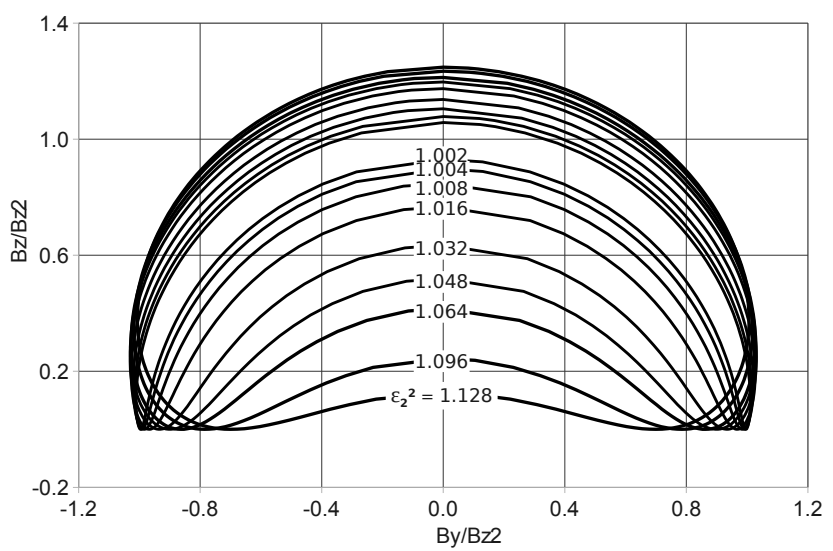

Fig. B1. Magnetic hodograms similar to Fig. 1, but for a fixed field rotation and larger values of $\epsilon_{2}^{2}=\left(A_{\mathrm{x} 2}^{2}-1\right)$. Parameter values used are $\gamma=2$ and $\beta_{2}=1$, which gives $u_{2}=1$ regardless of $\theta_{2}$.

$A_{\mathrm{x} 2}=1.001, \gamma=2, \beta_{2}=1$, and $\sin ^{2} \theta_{2}=0.996$. The exact values are given in Sect. 2.5 of Paper I. In Table A1, they are compared to the values from Eqs. (A1) and (A2) and also to those from Eqs. (1) and (2).

\section{Appendix B}

In this appendix, we show properties of the exact solution of the Hall-MHD equations given in Paper I, for cases where the solution involves large variations of the magnetic field magnitude. Such solutions are obtained by use of values of $\epsilon_{2}^{2}=\left(A_{\mathrm{x} 2}^{2}-1\right)$ that are no longer small. They may be relevant to the much narrower structures, comprising the magnetopause current layer, or parts of it, during ongoing magnetic field reconnection. Figure B1 shows a series of magnetic hodograms, representing these solutions for $\Delta \Psi^{*}=180^{\circ}$ and increasing values of $\epsilon_{2}^{2}$. It is seen from the figure that the ionpolarized discontinuity (the lower branch of the hodogram) can be made to exhibit a deep field minimum in its middle, while the electron-polarized discontinuity (the upper branch) shows a more modest maximum. Note that the parameter values $\left(\gamma=2, \beta_{2}=1, \sin ^{2} \theta_{2}=0.996\right)$ used in the figure are the same as those in Fig. 1. Note also that, as $\epsilon_{2}^{2}$ increases, the derivative $d B_{\mathrm{y}} / d x$ at the turning points becomes larger so
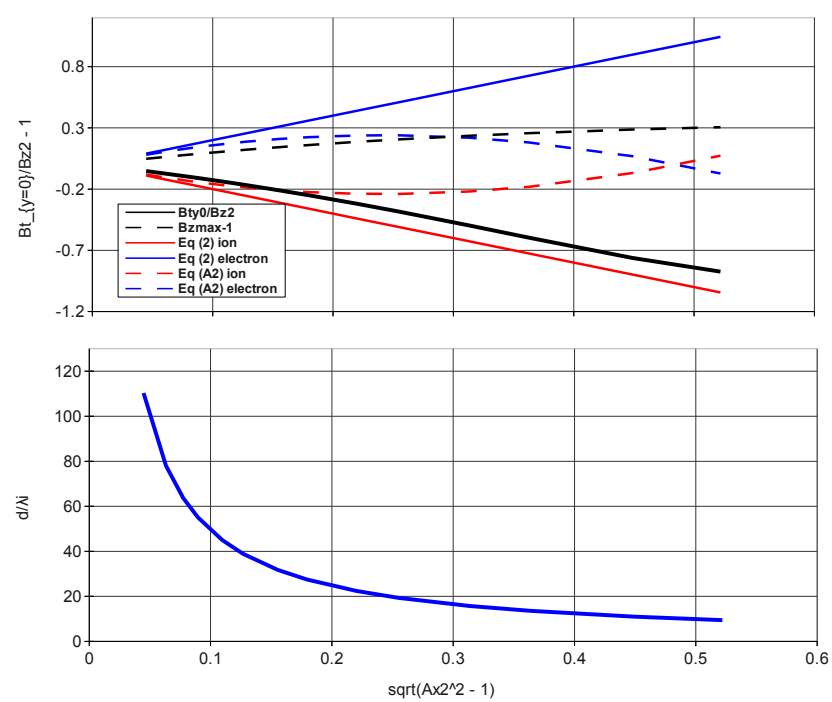

Fig. B2. Top panel: maximum field deviation, $\boldsymbol{B}_{\mathrm{dev}}-1=\boldsymbol{B}_{t 0} / B_{\mathrm{z} 2}-$ 1 as a function of $\epsilon_{2}=\sqrt{A_{\mathrm{x} 2}^{2}-1}$. Results from Fig. B1 are shown as solid curves, theoretical values from Eq. (2) as dashed lines, and results from Eq. (A2) as dotted curves. Red color indicates ion polarization, blue color electron polarization. Bottom panel: discontinuity thickness (in units of the ion inertial length) as a function of $\epsilon_{2}$. Red curves are for ion polarization, blue for electron polarization. The grey curve is the prediction from Eq. (5). Parameter values used are the same as in Fig. B1.

that these points become increasingly removed from being true fixed points of the system.

The top panel of Fig. B2 shows the maximum field deviation $\left(\boldsymbol{B}_{\mathrm{dev}}-1\right)=\left(\boldsymbol{B}_{t 0} / B_{\mathrm{z} 2}-1\right)$ in Fig. B1 as a function of $\epsilon_{2}=\sqrt{\left.A_{\mathrm{x} 2}^{2}-1\right)}$ as solid curves, and the prediction from Eq. (2) as dashed lines. Red curves refer to the ion polarization and blue to the electron polarization. Also shown are the predictions from Eq. (A2) as dotted curves in red or blue. The approximate formulas seem to work well for moderately small values of $\epsilon_{2}$. In the bottom panel of Fig. B2, solid curves show the behavior of the actual discontinuity thickness, $d / \lambda_{i 2}$, calculated from the integration in Eq. (15) of Paper I, again with red (blue) representing the ion (electron) polarization. Also shown by the grey curve is the prediction from Eq. (5). As expected, the thickness $d / \lambda_{i 2}$ initially decreases with increasing values of $\epsilon_{2}$, with nearly the same 
behavior for the electron- and ion-polarized branches. However, for larger values of $\epsilon_{2}$, the curves separate with the electron branch showing somewhat larger values of $d / \lambda_{\mathrm{i} 2}$. The curves in the figure are drawn for $u_{2}=1$. As can be seen from Eq. (5), the curves will give lower values of $d / \lambda_{i 2}$ for smaller values of $u_{2}$.

Acknowledgements. The research reported here results from a team effort, sponsored by the International Space Science Institute (ISSI), Bern, Switzerland, and devoted to the study of solar wind directional discontinuities. The data analysis has made use of the QSAS science analysis system, provided by the UK Cluster Science Centre (Imperial College, London, and Queen Mary University, London). We acknowledge the Cluster Active Archive and the CDAWeb for making the Cluster and ACE data available. We are greatly indebted to Thorsten Knetter for making his analysis results available to us.

The service charges for this open access publication have been covered by the Max Planck Society.

Topical Editor R. Nakamura thanks M. Neugebauer and another anonymous referee for their help in evaluating this paper.

\section{References}

Balogh, A., Dunlop, M. W., Cowley, S. W. H., Southwood, D. J., Thomlinson, J. G., Glassmeier, K. H., Musmann, G., Luhr, H., Buchert, S., Acuna, M. H., Fairfield, D. H., Slavin, J. A., Riedler, W., Schwingenschuh, K., and Kivelson, M. G.: The Cluster Magnetic Field Investigation, Space Sci. Rev., 79, 65-91, 1997.

Burlaga, L. F.: Directional Discontinuities in the Interplanetary Magnetic Field, Solar Phys., 7, 54-71, doi:10.1007/BF00148406, 1969.

Haaland, S. E., Sonnerup, B. U. Ö., Dunlop, M. W., Balogh, A., Georgescu, E., Hasegawa, H., Klecker, B., Paschmann, G., Puhl-Quinn, P., Rème, H., Vaith, H., and Vaivads, A.: Fourspacecraft determination of magnetopause orientation, motion and thickness: comparison with results from single-spacecraft methods, Ann. Geophys., 22, 1347-1365, doi:10.5194/angeo-221347-2004, 2004.

Hau, L.-N. and Sonnerup, B. U. Ö.: The structure of resistivedispersive intermediate shocks, J. Geophys. Res., 95, 1879118808, doi:10.1029/JA095iA11p18791, 1990.

Johnstone, A. D., Alsop, C., Burge, S., Carter, P. J., Coates, A. J., Coker, A. J., Fazakerley, A. N., Grande, M., Gowen, R. A., Gurgiolo, C., Hancock, B. K., Narheim, B., Preece, A., Sheather, P. H., Winningham, J. D., and Woodliffe, R. D.: Peace: a Plasma Electron and Current Experiment, Space Sci. Rev., 79, 351-398, doi:10.1023/A:1004938001388, 1997.

Khrabrov, A. V. and Sonnerup, B. U. Ö.: Error estimates for minimum variance analysis, J. Geophys. Res., 103, 6641-6652, doi:10.1029/97JA03731, 1998a.

Khrabrov, A. V. and Sonnerup, B. U. Ö.: DeHoffmann-Teller Analysis, in: Analysis Methods for Multi-Spacecraft Data, edited by: Paschmann, G. and Daly, P. W., no. SR-001 in ISSI Scientific Reports, available at: http://www.issibern.ch/publications/pdf/sr1. pdf, chap. 9, pp. 221-248, ESA Publ. Div., Noordwijk, Netherlands, 1998b.

Knetter, T.: A new perspective on the solar wind micro-structure due to multi-point observations of discontinuities, Ph.D. thesis, Univ. Köln, 2005.

Knetter, T., Neubauer, F. M., Horbury, T., and Balogh, A.: Fourpoint discontinuity observations using Cluster magnetic field data: A statistical survey, J. Geophys. Res., 109, A06102, doi:10.1029/2003JA010099, 2004.

Lee, L. C. and Kan, J. R.: Structure of the magnetopause rotational discontinuity, J. Geophys. Res., 87, 139-143, doi:10.1029/JA087iA01p00139, 1982.

Lepping, R. P. and Behannon, K. W.: Magnetic field directional discontinuities - Characteristics between 0.46 and 1.0 AU, J. Geophys. Res., 91, 8725-8741, doi:10.1029/JA091iA08p08725, 1986.

Neugebauer, M.: Anisotropy and Alfvénicity of hourly fluctuations in the fast polar solar wind, J. Geophys. Res., 109, A02101, doi:10.1029/2003JA009947, 2004.

Neugebauer, M.: Comment on the abundances of rotational and tangential discontinuities in the solar wind, J. Geophys. Res., 11, A04103, doi:10.1029/2005JA011497, 2006.

Paschmann, G. and Sonnerup, B. U. Ö.: Proper frame determination and Walén test, in: Multi-Spacecraft Analysis Methods Revisited, edited by: Paschmann, G. and Daly, P. W., no. SR-008 in ISSI Scientific Reports, available at: http://www.issibern.ch/ publications/pdf/sr8.pdf, chap. 7, pp. 65-74, ESA Communications, Noordwijk, Netherlands, 2008.

Rème, H., Bosqued, J. M., Sauvaud, J. A., Cros, A., Dandouras, J., Aoustin, C., Bouyssou, J., Camus, T., Cuvilo, J., Martz, C., Medale, J. L., Perrier, H., Romefort, D., Rouzaud, J., D‘Uston, C., Mobius, E., Crocker, K., Granoff, M., Kistler, L. M., Popecki, M., Hovestadt, D., Klecker, B., Paschmann, G., Scholer, M., Carlson, C. W., Curtis, D. W., Lin, R. P., McFadden, J. P., Formisano, V., Amata, E., Bavassano-Cattaneo, M. B., Baldetti, P., Belluci, G., Bruno, R., Chionchio, G., di Lellis, A., Shelley, E. G., Ghielmetti, A. G., Lennartsson, W., Korth, A., Rosenbauer, H., Lundin, R., Olsen, S., Parks, G. K., McCarthy, M., and Balsiger, H.: The Cluster Ion Spectrometry (CIS) Experiment, Space Sci. Rev., 79, 303-350, 1997.

Sonnerup, B. U. Ö. and Scheible, M.: Minimum and Maximum Variance Analysis, in: Analysis Methods for Multi-Spacecraft Data, edited by: Paschmann, G. and Daly, P. W., no. SR-001 in ISSI Scientific Reports, available at: http://www.issibern.ch/ publications/pdf/sr1.pdf, chap. 8, pp. 185-220, ESA Publ. Div., Noordwijk, Netherlands, 1998.

Sonnerup, B. U. O., Papamastorakis, I., Paschmann, G., and Luehr, H.: Magnetopause properties from AMPTE/IRM observations of the convection electric field - Method development, J. Geophys. Res., 92, 12137-12159, doi:10.1029/JA092iA11p12137, 1987.

Sonnerup, B. U. Ö., Haaland, S. E., and Paschmann, G.: On arcpolarized structures in the solar wind, Ann. Geophys., 28, 1229 1248, doi:10.5194/angeo-28-1229-2010, 2010.

Teh, W.-L., Sonnerup, B. U. Ö., Paschmann, G., and Haaland, S. E.: Local structure of directional discontinuities in the solar wind, J. Geophys. Res., 116, A04105, doi:10.1029/2010JA016152, 2011.

Tsurutani, B. T. and Smith, E. J.: Interplanetary discontinuities: Temporal variations and their radial gradient from 1 to $8 \mathrm{AU}, \mathrm{J}$ Geophys. Res., 84, 2773-2787, doi:10.1029/JA084iA06p02773, 
1979.

Vasquez, B. J. and Hollweg, J. V.: Formation of arc-shaped Alfvén waves and rotational discontinuities from oblique linearly polarized wave trains, J. Geophys. Res., 101, 13527-13540, doi:10.1029/96JA00612, 1996.

Vasquez, B. J., Abramenko, V. I., Haggerty, D. K., and Smith, C. W.: Numerous small magnetic field discontinuities of Bartels rotation 2286 and the potential role of Alfvénic turbulence, J. Geophys. Res. (Space Physics), 112, A11102, doi:10.1029/2007JA012504, 2007.
Vogt, J., Haaland, S., and Paschmann, G.: Accuracy of multi-point boundary crossing time analysis, Ann. Geophys., 29, 2239-2252, doi:10.5194/angeo-29-2239-2011, 2011.

Wu, B. H. and Lee, L. C.: Hall effects on the Walén relation in rotational discontinuities and Alfvén waves, J. Geophys. Res., 105, 18377-18390, doi:10.1029/2000JA900043, 2000. 